\title{
Thermal fluctuations in the conical state of monoaxial helimagnets
}

\author{
Victor Laliena, ${ }^{1, *}$ Yusuke Kato, ${ }^{2}$ Germán Albalate, ${ }^{1}$ and Javier Campo ${ }^{1, \dagger}$ \\ ${ }^{1}$ Instituto de Ciencia de Materiales de Aragón (CSIC-Universidad de Zaragoza), Calle Pedro Cerbuna 12, 50009 Zaragoza, Spain \\ ${ }^{2}$ Department of Basic Science, University of Tokyo, Meguro, Tokyo 153-8902, Japan
}

(Received 27 April 2018; revised manuscript received 17 July 2018; published 31 October 2018)

\begin{abstract}
The effect of thermal fluctuations on the phase structure of monoaxial helimagnets with external magnetic field parallel to the chiral axis is analyzed by means of a saddle point expansion of the free energy. The phase transition that separates the conical and forced ferromagnetic phases is changed to first order by the thermal fluctuations. In a purely monoaxial system the pitch of the conical state remains independent of temperature and magnetic field, as in mean-field theory, even when fluctuations are taken into account. However, in the presence of weak Dzyaloshinskii-Moriya interactions in the plane perpendicular to the chiral axis, thermal fluctuations induce a dependence of the pitch on temperature and magnetic field. This may serve to determine the nature of magnetic interactions in such systems.
\end{abstract}

DOI: 10.1103/PhysRevB.98.144445

\section{INTRODUCTION}

It is well known that the spin-orbit coupling originates antisymmetric bilinear exchange interactions between spins in crystalline structures that have no center of symmetry [1,2]. These so called Dzyaloshinskii-Moriya (DM) interactions are generically of the form $\vec{D}_{i j} \cdot\left[\vec{S}_{i} \times \vec{S}_{j}\right]$, where $\vec{S}_{i}$ represents the spin operator at site $i$ and $\vec{D}_{i j}$ is a vector that sets the strength of the interaction, and whose direction is dictated by the crystal symmetry. The DM interactions give rise to chiral modulated magnetic states such as helices, conical states, chiral soliton lattices, and skyrmion lattices [3-7]. Some of those chiral structures have been observed experimentally many years after the theoretical prediction [8-12], and are very promising candidates for spintronic and magnonic devices since the modulated magnetic structures appear spontaneously and can be controlled by externally imposed conditions, such as temperature and magnetic fields [13].

Systems in which $\vec{D}_{i j}$ is restricted to pairs of magnetic ions $i$ and $j$ that are aligned along a fixed crystalline direction, $\hat{D}$, called the DM axis, and $\vec{D}_{i j}=D \hat{D}$, are called monoaxial helimagnets. They are very interesting both from the fundamental physics, related to the realization and breaking of chiral symmetry, and from the practical point of view (spintronics) [14], and consequently much theoretical and experimental effort is being devoted to their study [10,14-36]. Monoaxial helimagnets include $\mathrm{CrNb}_{3} \mathrm{~S}_{6}$ and $\mathrm{Yb}\left(\mathrm{Ni}_{1-x} \mathrm{Cu}_{x}\right) \mathrm{Al}_{9}$ [14].

The direct experimental confirmation in 2012 by Togawa et al. [36] of the development of a chiral soliton lattice in $\mathrm{CrNb}_{3} \mathrm{~S}_{6}$, predicted long ago by Dzyaloshinskii in his pioneering work of 1965 [4], made this compound the archetypical monoaxial helimagnet. The other known monoaxial helimagnets, the series of compounds $\mathrm{Yb}\left(\mathrm{Ni}_{1-x} \mathrm{Cu}_{x}\right) \mathrm{Al}_{9}$, also have the chiral soliton lattice phase [37], but with a period much

\footnotetext{
*1aliena@unizar.es

†javier.campo@csic.es
}

shorter than in $\mathrm{CrNb}_{3} \mathrm{~S}_{6}$. Another difference with $\mathrm{CrNb}_{3} \mathrm{~S}_{6}$ is the lock-in of the chiral soliton lattice period as a function of magnetic field [37], which has been observed also in magnetoresistance for $x=0$ [38]. This lock-in has been attributed to itinerant heavy electrons [39].

The magnetic phase diagram of $\mathrm{CrNb}_{3} \mathrm{~S}_{6}$ has recently attracted much attention. Experiments show that with a magnetic field perpendicular to the DM axis a chiral modulated phase appears at low temperature and field. It is separated from a homogeneous forced FM (FFM) or PM phase by a line boundary in the temperature-magnetic field plane. The chiral phase is complex, with several crossovers that show the smooth evolution of a helical structure at low field to a chiral soliton lattice at higher field [16,25]. Measurements include magnetization curves, dc and ac susceptibilities, Lorenz microscopy, and magnetoresistance. The phase boundary is more complex than expected, with two lines of second-order transitions at low $T$ and high $T$ separated by an intermediate line of first-order transitions. Two tricritical points separate the first-order line from the two second-order lines [16]. These features of the phase boundary, including the presence of the tricritical points, were theoretically predicted before the experimental measurements were available [19-21]. The phase boundary can be understood in terms of the different nature of the phase transitions [20,40,41]. At low $T$ the second-order transition is of nucleation type, associated with the formation of a chiral soliton lattice; in contrast, at high $T$ the second-order phase transition is of instability type, corresponding to the appearance of helical order. These two lines of second-order transitions have thus a different nature and cannot merge smoothly. Hence, they are separated by an intermediate first-order line with one tricritical point at each end point.

Comparatively, the phase diagram with a magnetic field parallel to the DM axis has received little attention. Meanfield theory predicts a low $T$ and low field modulated conical state that propagates along the DM axis with a wave number independent of temperature and magnetic field. This phase 
is separated from a homogeneous FFM phase by a line of second-order transitions. The experimental results seem to confirm this simple picture, but the measurements are limited to magnetization curves and thus are not conclusive about the nature of the transition. To our knowledge, no measurement of the propagation wave number as a function of temperature and magnetic field has been reported.

From the theoretical point of view, the investigation of the effect of correlations in the thermal fluctuations, which may change some features of the phase diagram, has started only very recently [24]. In cubic helimagnets, it is known that thermal fluctuations modify the free energy of the different states in such a way that a metastable skyrmion lattice becomes the thermodynamical equilibrium state [11,40]. In monoaxial helimagnets it is not expected that fluctuations cause such dramatic effects, but the nature of the phase transitions and some features of the equilibrium states can be modified. Indeed, in Ref. [24], using the Green's function method, Masaki and Stamps reported an analysis of the role of fluctuations and anisotropies in the monoaxial helimagnet with a magnetic field applied along the DM axis. They concluded that the phase boundaries and the nature of the transitions are modified. In particular, they found metastability in the vicinity of the phase boundary and pointed out the possibility of a first-order phase transition.

In this paper we analyze the effect of thermal fluctuations in the monoaxial helimagnet in the presence of a magnetic field parallel to the DM axis via a saddle point expansion. The chiral modulated phase is a conical state. It is shown that thermal fluctuations change the nature of the conical to FFM phase transition from second to first order. It is also shown that the pitch of the conical phase, which is independent of magnetic field and temperature within the standard mean-field theory, where the correlations of fluctuations are neglected [19], acquires a dependence on magnetic field and temperature due to the fluctuations provided that chiral interactions of DM type are present in the plane perpendicular to the chiral axis. However, in a purely monoaxial helimagnet, with DM interaction restricted to a single axis, the pitch of the conical state remains independent of magnetic field and temperature even if fluctuations are taken into account. Therefore, the dependence of the pitch on the externally imposed conditions can be used to reveal weak magnetic interactions in monoaxial helimagnets.

The paper is organized as follows. In Sec. II we introduce the model and set the notation; Sec. III is devoted to a description of the saddle point method used to study the model; Sec. IV briefly analyzes the FFM state; Sec. V is devoted to the study of the conical state; in Sec. VI the results of the previous sections are applied to the purely monoaxial helimagnet; and in Sec. VII we study the effects of weak DM interactions in the plane perpendicular to the chiral axis. The paper ends with a brief summary and concluding remarks in Sec. VIII.

\section{MODEL}

We consider a classical spin system with exchange FM and DM interactions along three perpendicular axes, $\{\hat{x}, \hat{y}, \hat{z}\}$, and uniaxial single-ion magnetic anisotropy along an axis $\hat{u}$, that in principle can be either of easy-axis or easy-plane type. For simplicity, the FM interaction is taken isotropic in space, with strength $J$, but the DM interaction is different along the three different axes. In the continuum limit the energy is given by the effective Hamiltonian $\mathcal{H}=\epsilon_{0} \mathcal{W}$, where $\epsilon_{0}$ sets the energy scale and $\mathcal{W}$, a functional of the unit vector field $\hat{n}$ that represents the direction of the local magnetic moment, can be written as the integral of a density, $\mathcal{W}=\int d^{3} x W$, with

$$
\begin{aligned}
\frac{W}{q_{0}}= & \frac{1}{2} \sum_{i} \partial_{i} \hat{n} \cdot \partial_{i} \hat{n}+q_{0} \hat{n} \cdot \vec{D}_{\rho} \\
& \times \hat{n}-q_{0}^{2} \gamma(\hat{u} \cdot \hat{n})^{2}-q_{0}^{2} \vec{h} \cdot \hat{n} .
\end{aligned}
$$

In the above expression $\vec{D}_{\rho}=\sum_{i} \hat{x}_{i} \rho_{i} \partial_{i}$ is a differential operator, with $\partial_{i}=\partial / \partial x_{i}$, and $x_{i}$ runs over $\{x, y, z\}$ in the obvious way. The dimensionless coefficients $\rho_{i}$ are real numbers that set the relative strength of the DM interaction along each axis. The first term in (1) gives the FM exchange interaction; the second term represents the DM interaction, whose overall strength relative to the Heisenberg exchange interaction is given by $q_{0}$, which has the dimensions of inverse length; the third term corresponds to the single-ion anisotropy along the axis given by the unit vector $\hat{u}$, the intensity of which, relative to the Heisenberg exchange energy, is given by the dimensionless parameter $\gamma$, which introduces easy-axis anisotropy if it is positive and easy-plane anisotropy if it is negative; the last term is the Zeeman energy, and the dimensionless parameter $h$ is proportional to the magnetic field intensity. The continuum model is obtained from a lattice model, so that we have $q_{0}=J / D a_{\mathrm{L}}$ and $\epsilon_{0}=J S^{2} / q_{0} a$, where $J$ and $D$ are the Heisenberg and DM energies, $S$ the spin modulus, and $a_{\mathrm{L}}$ the lattice parameter along the DM axis [40]. Since $q_{0}$ sets the scale for the modulation of chiral magnetic states, the continuum approximation is justified if $q_{0} a_{\mathrm{L}} \ll 1$, that is, if the DM interaction is much weaker than the Heisenberg interaction.

Notice that $\vec{D}_{\rho}$ does not transform as a vector under rotations. In covariant notation the DM interaction has to be written as $\rho_{i j k} n_{i} \partial_{j} n_{k}$, where $\rho_{i j k}$ is a tensor antisymmetric under the exchange of $i$ and $k$, and summation over repeated indices is understood. Nevertheless, we find it convenient to work with the non-covariant notation. Hence, the equations presented in this paper hold in the reference frame in which $\rho_{i j k}=\rho_{i} \epsilon_{i j k}$, where $\epsilon_{i j k}$ is the totally antisymmetric tensor.

The cubic helimagnet is obtained if $\rho_{i}=1$ for all $i$, and the monoaxial helimagnet if $\rho_{x}=\rho_{y}=0$ and $\rho_{z}=1$. In the latter case the magnetic anisotropy should be directed along the same axis as the DM interaction, and therefore $\hat{u}=\hat{z}$.

The equilibrium properties of the system at temperature $T$ are given by the partition function,

$$
\mathcal{Z}=\int\left[d^{2} \hat{n}\right] \exp [-\mathcal{W} / t],
$$

where $t=T / T_{0}$ is a dimensionless temperature, with $T_{0}=$ $\epsilon_{0} / k_{\mathrm{B}}$.

To close this section, let us discuss the parameters appropriate for $\mathrm{CrNb}_{3} \mathrm{~S}_{6}[8,9]$, which has a noncentrosymmetric hexagonal crystal structure with space group $\mathrm{P}_{3} 22$. The lack of inversion symmetry allows a DM interaction propagating along the $c$ axis. A DM interaction in the plane 
perpendicular to $c$ is also allowed by the symmetry, provided that $\rho_{x}=\rho_{y}=\rho_{\mathrm{T}}$. The isotropy of the magnetic interactions in this plane is supported by the fact that no sensitivity of magnetization curves to the direction of the magnetic field component perpendicular to the $c$ axis has been found $[9,16]$. The zero-field critical temperature depends on the sample, and is $T_{\mathrm{c}} \approx 125 \mathrm{~K}$. The ordered phase is a helimagnet with a period $L_{0} \approx 480 \AA$, which corresponds to a wave number $q_{0}=0.013 \AA^{-1}$. The critical fields perpendicular and parallel to the $c$ axis depend also on the sample. They are of order 2000 Oe and 20000 Oe, respectively [9]. This big difference implies that there is an important easy-plane anisotropy described by the single-ion anisotropy term with $\hat{u}$ directed along the $c$ axis. The value $\gamma=-2.58$ is appropriate to reproduce the zero-temperature phase diagram [23]. The FM Heisenberg interaction is also spatially anisotropic, with the spins in the same $a b$ plane more strongly coupled than the spins in different $a b$ planes [21]. Thus, the high anisotropy suggests that $\rho_{\mathrm{T}} \ll \rho_{z}=1$, and the system is a monoaxial helimagnet. The experimental results (formation of a chiral soliton lattice when the magnetic field is perpendicular to $c$ ) are consistent with this view. The lattice parameter along the $c$ axis is $12.1 \AA$. The magnetic $\mathrm{Cr}$ ions, however, form an hcp lattice, with a separation between nearest-neighbor ions in the $a b$ plane of $5.73 \AA$, and of $6.90 \AA$ between nearest-neighbors in different $a b$ planes. The separation between $\mathrm{Cr}$ planes is, therefore, $a_{\mathrm{L}} \approx 6.05 \AA$. The continuum approach is justified since the period of the helimagnet is very large compared to the magnetic ion lattice parameter, $L_{0} / a_{\mathrm{L}} \approx 80$. To analyze the thermal fluctuations we use the short distance cutoff provided by the crystal structure, $\Lambda=\pi / a_{\mathrm{L}}=\left(L_{0} / 2 a_{\mathrm{L}}\right) q_{0}$, which it is $\Lambda \approx 40 q_{0}$ for $\mathrm{CrNb}_{3} \mathrm{~S}_{6}$.

\section{SADDLE POINT EXPANSION}

The inverse of the dimensionless temperature, $1 / t$, is a large number if $T \ll T_{0}$, and the partition function can be obtained by the saddle point expansion [40]. Consider the generic model defined by the energy density (1) and let $\hat{n}_{0}$ be a stationary point, that is, a solution of the Euler-Lagrange equations, $\delta \mathcal{W} / \delta \hat{n}=0$, which reads

$\nabla^{2} \hat{n}_{0}-2 q_{0}\left(\vec{D}_{\rho} \times \hat{n}_{0}\right)+2 q_{0}^{2} \gamma\left(\hat{u} \cdot \hat{n}_{0}\right) \hat{u}+q_{0}^{2} \vec{h}=\mu \hat{n}_{0}$,

where $\mu$ is a position-dependent Lagrange multiplier that implements the constraint $\hat{n}_{0}^{2}=1$, which supplements Eq. (3). Notice that the FFM state, with constant $\hat{n}_{0}$, is always a solution of the Euler-Lagrange equations.

The field $\hat{n}$ in the neighborhood of $\hat{n}_{0}$ can be written in terms of two real fields $\xi_{\alpha}(\alpha=1,2)$ as

$$
\hat{n}=\sqrt{1-\xi^{2}} \hat{n}_{0}+\sum_{\alpha} \xi_{\alpha} \hat{e}_{\alpha},
$$

where the three unit vectors $\left\{\hat{e}_{1}, \hat{e}_{2}, \hat{n}_{0}\right\}$ form a right-handed orthonormal triad. They can be parametrized in terms of the two angles $\theta$ and $\psi$ (determined by $\hat{n}_{0}$ ) as

$$
\begin{aligned}
& \hat{e}_{1}=(\cos \theta \cos \psi, \cos \theta \sin \psi,-\sin \theta), \\
& \hat{e}_{2}=(-\sin \psi, \cos \psi, 0), \\
& \hat{n}_{0}=(\sin \theta \cos \psi, \sin \theta \sin \psi, \cos \theta) .
\end{aligned}
$$

Let us expand $\mathcal{W}$ in powers of $\xi_{\alpha}$ up to quadratic order:

$$
\mathcal{W}=\mathcal{W}\left(\hat{n}_{0}\right)+\frac{q_{0}}{2} \int d^{3} x \sum_{\alpha, \beta} \xi_{\alpha} K_{\alpha \beta} \xi_{\beta}+O\left(\xi^{3}\right),
$$

with

$$
\begin{aligned}
K_{\alpha \beta}= & -\left[\nabla^{2}+2 W\left(\hat{n}_{0}\right) / q_{0}+q_{0}^{2} \vec{h} \cdot \hat{n}_{0}\right] \delta_{\alpha \beta}+\partial_{i} \hat{e}_{\alpha} \cdot \partial_{i} \hat{e}_{\beta} \\
& +q_{0}\left(\hat{e}_{\alpha} \cdot \vec{D}_{\rho} \times \hat{e}_{\beta}+\hat{e}_{\beta} \cdot \vec{D}_{\rho} \times \hat{e}_{\alpha}\right) \\
& -2 q_{0}^{2} \gamma\left(\hat{u} \cdot \hat{e}_{\alpha}\right)\left(\hat{e}_{\beta} \cdot \hat{u}\right)-(2 \vec{G} \cdot \nabla+\nabla \cdot \vec{G}) \epsilon_{\alpha \beta},
\end{aligned}
$$

where $\epsilon_{\alpha \beta}$ is the two-dimensional antisymmetric unit tensor,

$$
\vec{G}=\sum_{i}\left(\hat{e}_{1} \cdot \partial_{i} \hat{e}_{2}+q_{0} \rho_{i} \hat{x}_{i} \cdot \hat{n}_{0}\right) \hat{x}_{i},
$$

and $W(\hat{n})$ is given by Eq. (1). The linear term in Eq. (8) vanishes on account of the Euler-Lagrange equations.

The fluctuation operator $K_{\alpha \beta}$ is a symmetric differential operator that is positive definite if $\hat{n}_{0}$ is a local minimum of $\mathcal{W}$. In this case the free energy density, $f=-(t / V) \ln \mathcal{Z}$, can be obtained from the saddle point method [42], which is an asymptotic expansion in powers of $t$ that to lowest order, ignoring some irrelevant constants, gives

$$
f=\frac{1}{V} \mathcal{W}\left(\hat{n}_{0}\right)+(t / V) \ln \sqrt{\operatorname{det} K K_{0}^{-1}}+O\left(t^{2}\right) .
$$

The constant operator $K_{0 \alpha \beta}=-\nabla^{2} \delta_{\alpha \beta}$ is introduced merely as a convenient way of normalizing the contribution of fluctuations to the free energy. In the quantum field theory jargon, the first term of (11) is called the tree level and the term proportional to $t^{n}$ the $n$-loop order. If $K$ is not positive definite the stationary point is unstable and the saddle point expansion does not exist.

The 1-loop term diverges in the continuum limit due to the short-distance fluctuations and a short-distance cutoff is necessary. In solid state physics it is naturally provided by the crystal lattice. The fluctuation free energy is dominated by the short-distance fluctuations and depends strongly on the cutoff [11]. Hence, the comparison of free energies of states computed with different cutoff schemes (different lattice discretization) is not meaningful. The low-lying spectrum of $K$, however, is well defined in the continuum limit and shows a weak dependence on the cutoff.

The 1-loop approximation is valid if the terms of order $\xi^{3}$ and higher that are neglected in (8) do not give a large contribution. Since the leading contribution of the cubic term vanishes by symmetry, the contribution of the higher order terms relative to the quadratic terms can be estimated by the ratio $\left\langle\xi^{4}\right\rangle /\left\langle\xi^{2}\right\rangle \sim\left\langle\xi^{2}\right\rangle=t \operatorname{Tr} K^{-1} / q_{0} V$.

It is well known that critical fluctuations are generically non-Gaussian, and the 1-loop approximation does not describe the physics of critical points. The fluctuations considered in this work, however, are not critical. They become critical when the gap, $A$, vanishes and the soft modes become massless. As we will see in the next sections, this regime will not be approached in our computations. Thus, the role of fluctuations is merely to modify the free energy of the competing states, although this leads to changes in the properties of the equilibrium states and in the nature of the transitions. 


\section{FORCED FERROMAGNETIC STATE}

In this and the remaining sections we take the single-ion anisotropy and the magnetic field along $\hat{z}$, so that $\hat{u}=\hat{z}$ and $\hat{h}=h \hat{z}$. With no loss we take $h \geqslant 0$.

The FFM state is always a stationary point, with $\theta=0$ and $\psi$ undetermined (may be taken as $\psi=0$ ). Its $K$ operator,

$$
K_{\alpha \beta}=\left[-\nabla^{2}+q_{0}^{2}(h+2 \gamma)\right] \delta_{\alpha \beta}-2 \rho_{z} q_{0} \partial_{z} \epsilon_{\alpha \beta},
$$

is readily diagonalized by Fourier transform, and its spectrum reads

$$
\lambda_{ \pm}=k_{x}^{2}+k_{y}^{2}+\left(k_{z} \pm \rho_{z} q_{0}\right)^{2}+q_{0}^{2}\left(h+2 \gamma-\rho_{z}^{2}\right),
$$

where $\vec{k}$ is the wave vector of the eigenfunction. The lowest eigenvalue is attained for $k_{x}=k_{y}=0$ and $k_{z}= \pm \rho_{z} q_{0}$ and reads $\lambda_{\min }=\left(h+2 \gamma-\rho_{z}^{2}\right) q_{0}^{2}$. Therefore, the FFM state is stable for $h>h_{\mathrm{c}}$ and unstable for $h<h_{\mathrm{c}}$, where

$$
h_{\mathrm{c}}=\rho_{z}^{2}-2 \gamma
$$

is the tree-level (mean field) critical field.

\section{CONICAL STATE}

The conical state, which has the form $\theta=\theta_{0}$ and $\psi=q z$, where $\theta_{0}$ and $q$ are constants, is a stationary state for any value of the $\rho_{i}$. The Euler-Lagrange equations are satisfied if and only if the relation

$$
\cos \theta_{0}=\frac{h}{h_{\mathrm{c}}-\Delta^{2}(q)}
$$

holds, where

$$
\Delta(q)=q / q_{0}-\rho_{z} .
$$

Since $\left|\cos \theta_{0}\right| \leqslant 1$, this stationary point exists only for

$$
\Delta^{2} \leqslant h_{c}-h
$$

This equation sets bounds to the pitch of the conical state, $q$, and implies also $0 \leqslant h \leqslant h_{\mathrm{c}}$. A second possibility for Eq. (15) is $\Delta^{2}>h_{\mathrm{c}}+h$, which implies that the mean magnetic moment is opposite to the applied magnetic field, lies in the unstable region, and need not be considered.

The tree-level free energy of the conical state is a function of the wave number $q$ :

$$
W_{\mathrm{C}}(\Delta)=\frac{q_{0}^{2}}{2}\left[\Delta^{2}-\rho_{z}^{2}-\frac{h^{2}}{h_{\mathrm{c}}-\Delta^{2}}\right] .
$$

The equilibrium value of $q$ is determined by minimizing the free energy in the region where the stationary point is locally stable. The minimum is attained at $\Delta=0$, and thus the equilibrium value is $q_{\mathrm{eq}}=\rho_{z} q_{0}$, which is independent of $\rho_{x}, \rho_{y}$, i.e., of the DM interaction in the transverse plane $X Y$, and of the magnetic field $h$ and the strength of the uniaxial anisotropy, $\gamma$.

The fluctuation operator can be readily obtained:

$$
\begin{gathered}
K_{11}=-\nabla^{2}+q_{0}^{2} A, \\
K_{22}=-\nabla^{2}, \\
K_{12}=-2 q_{0} \sin \theta_{0}\left(\rho_{x} \cos q z \partial_{x}+\rho_{y} \sin q z \partial_{y}\right) \\
+2 q_{0} \Delta \cos \theta_{0} \rho_{z} \partial_{z},
\end{gathered}
$$

where

$$
A=h_{\mathrm{c}}-\Delta^{2}-h^{2} /\left(h_{\mathrm{c}}-\Delta^{2}\right)
$$

is a constant. Notice that $A$ is positive in the neighborhood of $\Delta=0$ owing to the inequality (17). However, it is negative if $\Delta^{2}>h_{\mathrm{c}}+h$. In Appendix A it is shown that the operator $K$ is positive definite for $\Delta=0$ if $A_{0} \geqslant 0$ and $\rho_{\mathrm{m}}^{2}<h_{\mathrm{c}}$, where $A_{0}$ is the value of $A$ at $\Delta=0$ and $\rho_{\mathrm{m}}=\max \left\{\left|\rho_{x}\right|,\left|\rho_{y}\right|\right\}$. Thus the conical state is a locally stable stationary state if the DM interaction in the plane perpendicular to the propagation direction is weak enough.

In the remaining of the paper we restrict our attention to nearly monoaxial helimagnets, in which the DM interactions in the plane perpendicular to the chiral axis, $\hat{z}$, are much weaker than along this axis. For simplicity, we consider isotropic interactions within the perpendicular plane, so that $\rho_{x}=\rho_{y}=\rho_{\mathrm{T}} \ll \rho_{z}$. With no loss we set $\rho_{z}=1$.

For a nearly monoaxial helimagnet, the 1-loop free energy of the conical state can be obtained perturbatively by an expansion in powers of $\rho_{\mathrm{T}}$. The fluctuation operator can be written as $K=K^{(0)}+\rho_{\mathrm{T}} Q$, where $K^{(0)}$ corresponds to the monoaxial helimagnet, given by setting $\rho_{x}=\rho_{y}=0$ in Eqs. (19)-(21), and

$$
Q_{\alpha \beta}=-2 q_{0} \sin \theta_{0}\left(\cos q z \partial_{x}+\sin q z \partial_{y}\right) \epsilon_{\alpha \beta} .
$$

The 1-loop free energy can be expanded in powers of $Q$ as follows:

$$
\ln \operatorname{det} K=\operatorname{Tr} \ln K^{(0)}-\sum_{n=1}^{\infty} \frac{(-1)^{n}}{n} \rho_{\mathrm{T}}^{n} \operatorname{Tr}\left(Q K^{(0)^{-1}}\right)^{n} .
$$

Then, the free energy to 1-loop order can be written as

$$
f(\Delta)=W_{\mathrm{C}}(\Delta)+t\left[I_{0}(\Delta)-\sum_{n=1}^{\infty}(-1)^{n} \rho_{\mathrm{T}}^{n} I_{n}(\Delta)\right],
$$

where

$$
I_{0}(\Delta)=\frac{1}{2 V} \operatorname{Tr} \ln \left(K^{(0)} K_{0}^{-1}\right)
$$

and, for $n \geqslant 1$,

$$
I_{n}(\Delta)=\frac{1}{2 n V} \operatorname{Tr}\left(Q K^{(0)^{-1}}\right)^{n} .
$$

These functions are studied in Appendix $\mathrm{C}$ for $n \leqslant 2$. It happens that $I_{1}(\Delta)$ vanishes. Some of these functions, for instance $I_{0}$, are ultraviolet divergent and thus a short-distance cutoff has to be introduced. For the numerical evaluation we use a sharp cutoff in the wave vectors, $|\vec{k}|<\Lambda$, with $\Lambda / q_{0}=$ 40, a value appropriate for $\mathrm{CrNb}_{3} \mathrm{~S}_{6}$ (see Sec. II).

\section{MONOAXIAL HELIMAGNET}

In the previous section it has been shown that the tree-level equilibrium period of the conical state is $q_{\mathrm{eq}}=\rho_{z} q_{0}$, independently of magnetic field and the other parameters of the model. Indeed, the tree-level free energy is an even function of $\Delta$ and thus $\Delta=0$ has to be either a maximum or a minimum. It turns out that it is always a minimum in the region of stability of the conical state. It was shown in Ref. [40] that in cubic helimagnets the 1-loop fluctuations induce a dependence of the 
conical state wave number on magnetic field and temperature, due to the fact that the spectrum of its fluctuation operator is not invariant under the change of $\Delta$ by $-\Delta$ and, therefore, the 1-loop free energy shifts the minimum away from $\Delta=0$. The same is expected for generic noncubic helimagnets.

For the monoaxial helimagnet, however, the spectrum of the conical state fluctuation operator is invariant under the exchange of $\Delta$ by $-\Delta$, since $K_{\alpha \beta}^{(0)}(-\Delta)=K_{\beta \alpha}^{(0)}(\Delta)$. Thus, at least for low enough $t$, the free energy minimum is not shifted from $\Delta=0$ and the equilibrium wave number of the conical state is constant, independently of magnetic field and temperature.

Let us analyze the stability of the monoaxial helimagnet in detail. The spectrum of $K^{(0)}$ is studied in Appendix B. Its eigenfunctions are plane waves with wave vector $\vec{k}$ and eigenvalues $\lambda_{\sigma}(\vec{k})$, with $\sigma= \pm 1$, whose expression is given in Eqs. (B3) and (B4). Hence, the spectrum of $K$ contains two branches. The $\sigma=+1$ branch has a gap equal to $A$. The $\sigma=-1$ branch is gapless and corresponds to a Goldstone boson associated to the spontaneous breaking of rotational symmetry in spin space around the magnetic field direction. The presence of the Goldstone modes does not invalidate the saddle point expansion, since the interactions of the Goldstone modes vanish at zero momentum [43], so that the contribution of the zero mode to the higher order terms of the saddle point expansion vanish. Therefore, the validity of the 1-loop approximation at low enough $t$ is guaranteed by the gap, $A$.

The Goldstone branch becomes unstable for large $\Delta$. To see this, notice that $\lambda_{-}$is an even function of $k_{z}$ that for $k_{x}=$ $k_{y}=0$ has the following expansion in powers of $k_{z}$ :

$$
\lambda_{-}\left(k_{z}\right)=\left(1-\frac{4 \Delta^{2} \cos ^{2} \theta_{0}}{A}\right) k_{z}^{2}+O\left(k_{z}^{4}\right)
$$

Hence, $\lambda_{-}$becomes negative if $4 \Delta^{2} \cos ^{2} \theta_{0} / A>1$. Thus, the conical state becomes unstable for $|\Delta|>\Delta_{i}$ where $\Delta_{i}$ is the solution of

$$
\frac{4 \Delta_{i}^{2} \cos ^{2} \theta_{0}}{A}=1,
$$

where $\cos \theta_{0}$ and $A$ are functions of $\Delta_{i}$. The above equation is cubic in $\Delta_{i}$ and can be solved analytically, but we do not write the explicit solution here. It happens that $\Delta_{i}^{2}<h_{\mathrm{c}}-h$, and thus the conical state exists as a stationary point for $\Delta^{2}<$ $h_{\mathrm{c}}-h$, but it is stable only if $\Delta^{2}<\Delta_{i}^{2}$, while for $\Delta_{i}^{2}<\Delta^{2}<$ $h_{\mathrm{c}}-h$ is unstable. For $h_{\mathrm{c}}-h<\Delta^{2}<h_{\mathrm{c}}+h$ the conical state does not exist, owing to Eq. (15), and for $\Delta^{2}>h_{\mathrm{c}}+h$ the conical state exists but it is unstable. The same behavior was found in the cubic case [40]. Obviously, the free energy of Gaussian (1-loop) fluctuations is meaningful only for $|\Delta|<\Delta_{i}$.

The free energy to 1-loop order is given by setting $\rho_{\mathrm{T}}=0$ in Eq. (25). It happens that $I_{0}(\Delta)$ is an even function of $\Delta$ that has the opposite sign of $W_{C}(\Delta)$. Thus, there is a competition between the tree-level and 1-loop components of the free energy. Figure 1 displays $W_{\mathrm{C}}$ and $I_{0}$ as a function of $\Delta$ for $\gamma=-2.58$ and $h=4$, with a cutoff in wave numbers $\Lambda / q_{0}=40$. Notice that $I_{0}(\Delta)$ remains finite and well defined in the limits $\Delta \rightarrow \pm \Delta_{i}$.

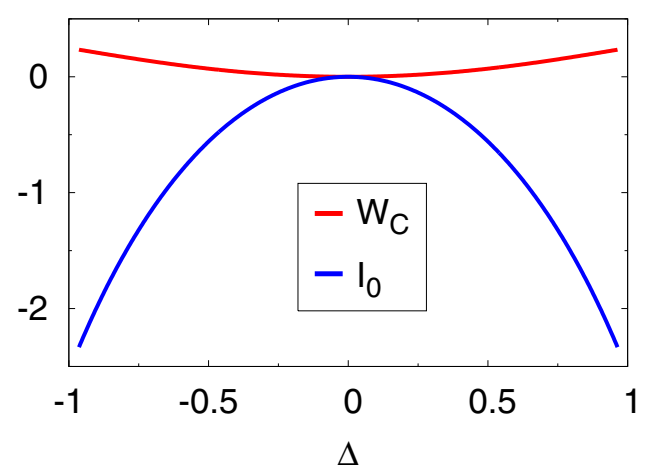

FIG. 1. Components of the free energy (tree level, $W_{\mathrm{C}}$, and 1loop, $\left.I_{0}\right)$ as a function of $\Delta$ for $\gamma=-2.58$ and $h=4$.

A phase transition results from the competition between $W_{\mathrm{C}}$ and $I_{0}$. For low $t$ the tree level dominates the free energy and its minimum is at $\Delta=0$. At a critical temperature, $t=$ $t_{\mathrm{c} 1}$, the local minimum at $\Delta=0$ equals the free energy at the limiting value $\Delta_{i}$ :

$$
t_{\mathrm{c} 1}=-\frac{W_{\mathrm{C}}\left(\Delta_{i}\right)-W_{\mathrm{C}}(0)}{I_{0}\left(\Delta_{i}\right)-I_{0}(0)} .
$$

A first-order phase transition takes place at $t_{\mathrm{c} 1}$. The conical state remains metastable for $t_{\mathrm{c} 1}<t<t_{\mathrm{c} 2}$, where

$$
t_{\mathrm{c} 2}=-\frac{W_{\mathrm{C}}^{\prime \prime}(0)}{I_{0}^{\prime \prime}(0)}
$$

is the temperature at which $\Delta=0$ becomes a maximum of the free energy. For $t>t_{\mathrm{c} 2}$ the conical state is not even metastable. In our numerical example we obtained $t_{\mathrm{c} 1}=$ 0.1002 and $t_{\mathrm{c} 2}=0.1339$. The behavior of the free energy by increasing temperature is illustrated in Fig. 2 for the case $h=4.0$.

Notice that the first-order phase transition takes place before the gap vanishes and thus before the fluctuations become critical. Indeed, critical fluctuations are characterized by the presence of infrared divergences that invalidate the Gaussian (1-loop) approximation, but no infrared divergence appears in our computation.

The phase diagram is displayed in Fig. 3. The temperature is normalized by the zero-field critical temperature, $T_{\mathrm{C}}$. With our choice of parameters for the numerical computations we have $T_{\mathrm{C}}=0.462 T_{0}$. The red line represents the transition line, given by $t_{\mathrm{c} 1}$. The conical state is metastable in the region filled by stripes, and disappears on the blue line, $t_{\mathrm{c} 2}$. The criterion that the saddle point expansion is valid if $\left\langle\xi^{2}\right\rangle \lesssim 0.1$ is satisfied on the left-hand side of the vertical black line. Thus, the solid lines of the $t_{\mathrm{c} 1}$ and $t_{\mathrm{c} 2}$ boundaries are reliable, while the broken lines may receive important contributions from higher order terms and we do not consider them reliable.

The pink line signals, for comparison, the phase boundary obtained with the variational mean field approach, which predicts a second-order instability type phase transition [19]. The saddle point expansion is reliable at low temperature, but fails at high temperature. The variational mean field theory is the lowest order term of a cumulant expansion, and neglects the correlations between the spin fluctuations at different sites. No small parameter justifies this expansion and it is thus 

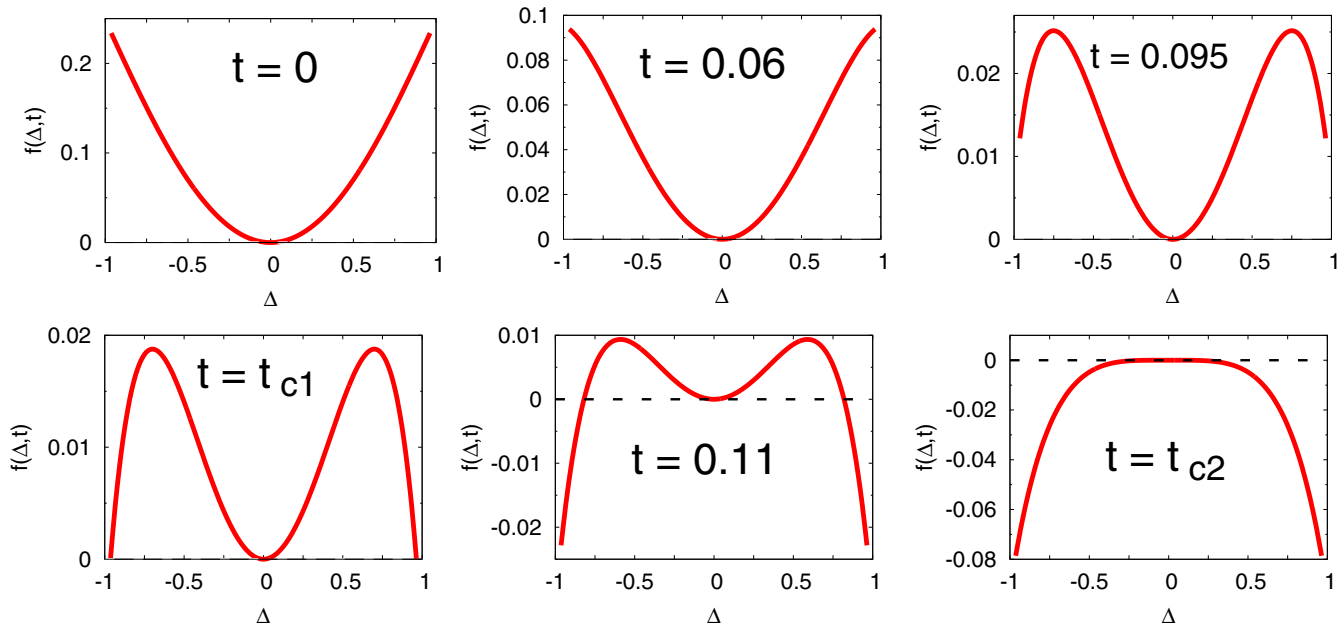

FIG. 2. The behavior of the free energy as a function of $\Delta$ for different temperatures and fixed $h=4.0$, in the pure monoaxial case $\left(\rho_{\mathrm{T}}=0\right)$.

questionable, although it is more reliable at higher temperatures, since the correlations between fluctuations diminish as temperature increases. The exception, of course, is the zero-field critical point, where the fluctuations are strongly correlated. The conclusion is then that the phase transition is of first order at low temperature, as predicted by the saddle point expansion, and of second-order instability type at high temperature, as predicted by the variational mean field theory. These two transitions of different nature have to be separated by a tricritical point. Thus the phase diagram obtained in Ref. [19] from the variational mean field theory has to be modified at low temperature.

The emergence of first-order transitions originated by fluctuations is reminiscent of the Coleman-Weinberg mechanism in quantum field theory [44] and the related HalperinLubensky-Ma effect in superconductors and liquid crystals

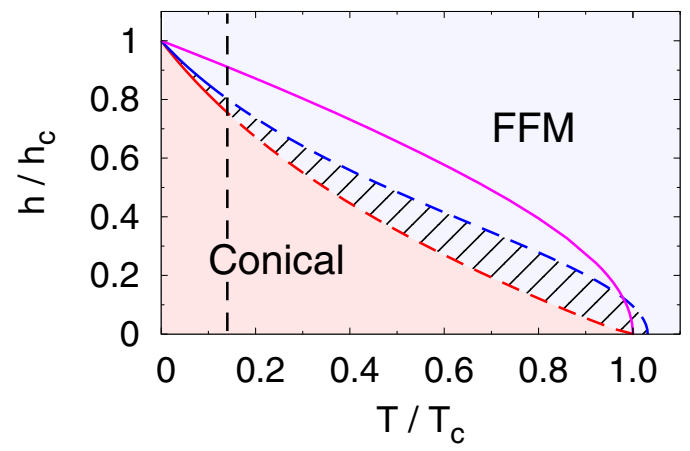

FIG. 3. Phase diagram of the monoaxial helimagnet in the Gaussian approximation. The conical state is stable on the red region and metastable in the striped region. The red line corresponds to first-order phase transitions $\left(t_{\mathrm{c} 1}\right)$. The metastable conical state disappears on the blue line $\left(t_{\mathrm{c} 2}\right)$. The 1-loop (Gaussian) approximation is considered reliable on the left-hand side of the vertical dashed line. The pink line is the phase boundary predicted by the variational mean field approximation. The transition in this case is of second-order instability type. The temperature is normalized to the zero-field transition temperature, $T_{\mathrm{C}}$.
[45]. In these cases Gaussian fluctuations modify the effective potential and drive the phase transition from second to first order.

\section{NEARLY MONOAXIAL HELIMAGNET}

In this section it is shown that the equilibrium period of the conical state of a nearly monoaxial helimagnet shows a weak dependence on magnetic field and temperature, proportional to $\rho_{\mathrm{T}}^{2}$, since thermal fluctuations at the 1-loop level shift the free energy minimum away from $\Delta=0$. Thus, any variation of the conical state period with temperature or field of a presumed monoaxial helimagnet reveals weak chiral interactions in the plane perpendicular to the chiral axis.

The equilibrium value of $\Delta$, denoted by $\Delta_{\text {eq }}$, corresponds to the minimum of the free energy, so that it obeys the equation

$$
W_{\mathrm{C}}^{\prime}\left(\Delta_{\mathrm{eq}}\right)+t\left[I_{0}^{\prime}\left(\Delta_{\mathrm{eq}}\right)-\rho_{\mathrm{T}}^{2} I_{2}^{\prime}\left(\Delta_{\mathrm{eq}}\right)\right]=0,
$$

where the prime stands for the derivative with respect to $\Delta$.

Notice that $W_{\mathrm{C}}$ and $I_{0}$ are even functions of $\Delta$, and it has been shown in the previous section that the free energy minimum is always at $\Delta=0$ if $\rho_{\mathrm{T}}=0$. For small $\rho_{\mathrm{T}}$ the equilibrium value of $\Delta$ will be of order $\rho_{\mathrm{T}}^{2}$ and can be expressed as

$$
\Delta_{\mathrm{eq}}=\rho_{\mathrm{T}}^{2} \Upsilon(t, h) .
$$

Expanding Eq. (32) around $\Delta_{\text {eq }}=0$ we get

$$
\Upsilon(t, h)=\frac{t I_{2}^{\prime}(0)}{W_{\mathrm{C}}^{\prime \prime}(0)+t I_{0}^{\prime \prime}(0)} .
$$

The equilibrium wave number of the conical state is given by

$$
\frac{q_{\mathrm{eq}}}{q_{0}}=\rho_{z}+\rho_{\mathrm{T}}^{2} \Upsilon(t, h) .
$$

The function $\Upsilon(t, h)$ is plotted as a function of $h$ for several values of $t$ in Fig. 4. Notice that $\Upsilon(t, h)$ is negative and decreases with $h$. Thus the wave number decreases (and the period increases) with temperature and magnetic field. This is consistent with the fact that the FFM state will be attained by increasing temperature or magnetic field. The solid and 


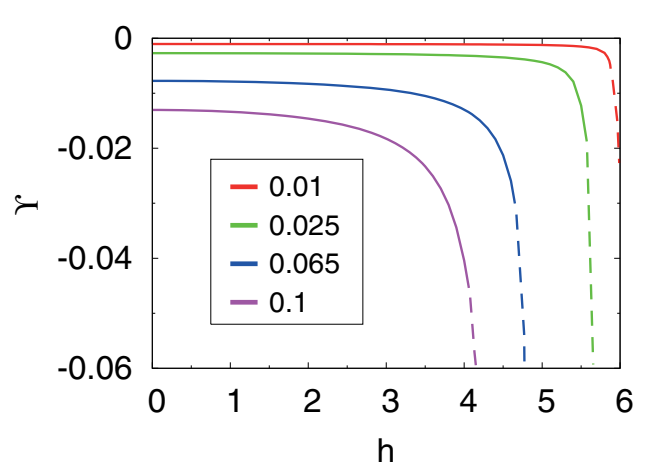

FIG. 4. The wave number variation with $h$, given by the function $\Upsilon(t, h)$, for the values of the dimensionless temperature, $t$, displayed in the legend. Solid and dashed lines correspond to the regions where the conical state is stable and metastable, respectively (red and striped regions of Fig. 3).

dashed lines of Fig. 4 correspond respectively to the regions in which the conical state is stable and metastable (i.e., to the red and striped regions of Fig. 3). Notice that $\Upsilon(t, h)$ diverges as $t \rightarrow t_{\mathrm{c} 2}$. This divergence, however, is spurious, since the perturbative computation assumes that $\Upsilon(t, h)$ is small and thus breaks down as it becomes large. Finally, notice that although the relative variation of the wave number is small, it grows with temperature, and it can become important at higher temperatures where the computations presented here are not valid.

\section{CONCLUSIONS}

Mean-field theory, which neglects the correlations between thermal fluctuations, predicts a second-order instability type phase transition between the conical and the FFM states in a monoaxial helimagnet with a magnetic field parallel to the chiral axis. In this paper we have shown that the correlation of fluctuations at Gaussian level, computed via the saddle point expansion to 1-loop order, changes the nature of the phase transition from second to first order. Signals of a firstorder transition for this system have also been noticed in Ref. [24], where the correlation of fluctuations are included via the Green's function method. The saddle point expansion, which is an asymptotic expansion in powers of $T / T_{0}$ and therefore not valid at high temperature, is more reliable at low temperature than the variational mean field theory. Therefore, it is likely that the transition changes from first to second order as temperature increases. This means that a tricritical point appears on the phase boundary.

It is worthwhile to point out that fluctuations of different types drive phase transitions to first order in different systems, from relativistic quantum fields [44] to superconductors and liquid crystals [45], and cubic helimagnets at low magnetic field [46,47].

According to mean-field theory, the pitch of the conical state of an helimagnet is independent of temperature and magnetic field. For a pure monoaxial helimagnet, with DM interactions only along one axis, the correlated fluctuations preserve this property. But in the presence of DM interactions in the plane perpendicular to the chiral axis, however weak they are, thermal fluctuations induce a dependence of the pitch on temperature and magnetic field. This variation of the pitch with the externally imposed conditions may be used to determine the nature of the chiral interactions in helimagnets. The perturbative computations presented here give only a small variation of the pitch in the region where they are reliable, but show a growing trend with temperature and thus it may be experimentally detectable. Thus, any temperature or field dependence of the pitch in presumed monoaxial chiral magnets, such as $\mathrm{CrNb}_{3} \mathrm{~S}_{6}$, can be interpreted as a departure from a purely monoaxial DM interaction.

\section{ACKNOWLEDGMENTS}

The authors thank Y. Masaki for fruitful discussions in the early stage of the collaboration. Grant No. MAT201568200-C2-2-P from the Spanish Ministry of Economy and Competitiveness, Grant No. 25220803 from the scientific JSPS Grant-in-Aid for Scientific Research (S), and Grant No. JP17H02923 from JSPS KAKENHI are acknowledged. This work was also supported by the MEXT program for promoting the enhancement of research universities, by the JSPS Core-to-Core Program, A (Advanced Research Networks), by the Chirality Research Center (Crescent) at Hiroshima University, and by JSPS and RFBR under the Japan-Russia Research Cooperative Program.

\section{APPENDIX A: LOCAL STABILITY OF THE CONICAL STATE}

Let us show that the conical state fluctuation operator, $K$, defined by Eqs. (19)-(21), with $\Delta=0$ is definite positive if $\rho_{x}$ and $\rho_{y}$ are small enough (here we do not assume isotropy in the plane perpendicular to the chiral axis). The most general square integrable wave function can be written as

$$
\xi_{\alpha}(\vec{x})=\int \frac{d^{3} k}{(2 \pi)^{3}} \exp (i \vec{k} \cdot \vec{x}) \tilde{\xi}_{\alpha}(z, \vec{k}),
$$

where $-q_{0} / 2 \leqslant k_{z} \leqslant q_{0} / 2$ and $\tilde{\xi}_{\alpha}(z, \vec{k})$ is periodic in $z$ :

$$
\tilde{\xi}_{\alpha}\left(z+L_{0}\right)=\tilde{\xi}_{\alpha}(z)
$$

with $L_{0}=2 \pi / q_{0}$. The expectation value of $K$ with this generic wave function is given by

$$
\langle\xi|K| \xi\rangle=\int \frac{d^{3} k}{(2 \pi)^{3}} \int_{0}^{L_{0}} d z T(z, \vec{k}),
$$

with

$$
\begin{aligned}
T(z, \vec{k})= & \sum_{\alpha}\left[k^{2}\left|\tilde{\xi}_{\alpha}\right|^{2}+\left|\tilde{\xi}_{\alpha}^{\prime}\right|^{2}+2 \operatorname{Im}\left(k_{z} \tilde{\xi}_{\alpha}^{*} \tilde{\xi}_{\alpha}^{\prime}\right)\right]+q_{0}^{2} A_{0}\left|\tilde{\xi}_{1}\right|^{2} \\
& +2 \operatorname{Im}\left[q _ { 0 } \operatorname { s i n } \theta _ { 0 } \left(\rho_{x} k_{x} \cos q_{0} z\right.\right. \\
& \left.\left.+\rho_{y} k_{y} \sin q_{0} z\right) \tilde{\xi}_{1}^{*} \tilde{\xi}_{2}\right],
\end{aligned}
$$

where $A_{0}=h_{c}-h^{2} / h_{c}$ is the value of $A$ at $\Delta=0$, the prime stands for derivative with respect to $z$, and we omit the arguments $z$ and $\vec{k}$ in the functions $\tilde{\xi}_{\alpha}$ and $\tilde{\xi}_{\alpha}^{\prime}$. Using the inequalities $a+b \geqslant a-|b|$, valid for any pair of real numbers $a$ and $b,|\operatorname{Im} c| \leqslant|c|$, valid for any complex number $c$, and

$$
\left|\rho_{x} k_{x} \cos q_{0} z+\rho_{y} k_{y} \sin q_{0} z\right| \leqslant \rho_{\mathrm{m}} k_{\mathrm{T}}
$$


where $\rho_{\mathrm{m}}=\max \left\{\left|\rho_{x}\right|,\left|\rho_{y}\right|\right\}$ and $k_{\mathrm{T}}^{2}=k_{x}^{2}+k_{y}^{2}$, we have

$$
\langle\xi|K| \xi\rangle \geqslant \int \frac{d^{3} k}{(2 \pi)^{3}} \int_{0}^{L_{0}} d z\left[\sum_{\alpha}\left(\left|k_{z} \tilde{\xi}_{\alpha}\right|-\left|\tilde{\xi}_{\alpha}^{\prime}\right|\right)^{2}+\Pi\right],
$$

where

$$
\Pi=k_{\mathrm{T}}^{2}\left|\tilde{\xi}_{2}\right|^{2}+\left(k_{\mathrm{T}}^{2}+q_{0}^{2} A_{0}\right)\left|\tilde{\xi}_{1}\right|^{2}-2 q_{0} \sin \theta_{0} \rho_{\mathrm{m}} k_{\mathrm{T}}\left|\tilde{\xi}_{1}\right|\left|\tilde{\xi}_{2}\right|
$$

is a quadratic form in $\left|\tilde{\xi}_{\alpha}\right|$. The operator $K$ will be positive definite if $\Pi$ is positive definite for any $k_{\mathrm{T}}$. The condition for the quadratic form to be positive definite is that all its principal minors be positive, that is,

$$
\begin{gathered}
k_{\mathrm{T}}^{2}+q_{0}^{2} A_{0} \geqslant 0, \\
k_{\mathrm{T}}^{2}\left(k_{\mathrm{T}}^{2}+q_{0}^{2} A_{0}-q_{0}^{2} \rho_{\mathrm{m}}^{2} \sin ^{2} \theta_{0}\right) \geqslant 0 .
\end{gathered}
$$

The first inequality implies $A_{0} \geqslant 0$, and the second inequality gives $\rho_{\mathrm{m}}^{2} \leqslant A_{0} / \sin ^{2} \theta_{0}$. Recalling the expressions for $A_{0}$ and $\sin ^{2} \theta_{0}$ we get

$$
\rho_{\mathrm{m}}^{2} \leqslant \rho_{z}^{2}-2 \gamma
$$

\section{APPENDIX B: SPECTRUM OF $K^{(0)}$}

The operator $K^{(0)}$, given by Eqs. (19)-(21) with $\rho_{x}=\rho_{y}=$ 0 , can be diagonalized by Fourier transform. Its normalized eigenfunctions are plane waves

$$
\left|\xi^{\sigma}(\vec{k})\right\rangle=\frac{1}{\sqrt{V}}\left(\begin{array}{l}
\phi_{1}^{\sigma}(\vec{k}) \\
\phi_{2}^{\sigma}(\vec{k})
\end{array}\right) e^{i \vec{k} \cdot \vec{x}}
$$

with $\sigma= \pm 1, V$ is the volume, and

$$
\phi_{1}^{\sigma *}(\vec{k}) \phi_{1}^{\sigma^{\prime}}(\vec{k})+\phi_{2}^{\sigma *}(\vec{k}) \phi_{2}^{\sigma^{\prime}}(\vec{k})=\delta_{\sigma \sigma^{\prime}} .
$$

They form a complete set. The corresponding eigenvalues are

$$
\lambda_{\sigma}(\vec{k})=k^{2}+f^{(\sigma)}\left(k_{z}\right),
$$

with

$$
f^{(\sigma)}\left(k_{z}\right)=\frac{q_{0}^{2} A}{2}\left(1+\sigma \sqrt{1+\frac{16 \Delta^{2} \cos ^{2} \theta_{0}}{A^{2}} \frac{k_{z}^{2}}{q_{0}^{2}}}\right) .
$$

The $\sigma=+1$ branch of the spectrum has a gap of value $q_{0}^{2} A$. The $\sigma=-1$ branch is gapless and corresponds to a Goldstone boson associated with the spontaneous breaking of the rotational symmetry in spin space corresponding to the rotation around the magnetic field direction. form

The polarization of the plane waves can be chosen of the

$$
\left(\begin{array}{l}
\phi_{1}^{\sigma}\left(k_{z}\right) \\
\phi_{2}^{\sigma}\left(k_{z}\right)
\end{array}\right)=\frac{1}{\sqrt{1+\Theta^{2}}}\left(\begin{array}{l}
(-i \Theta)^{\frac{1-\sigma}{2}} \\
(-i \Theta)^{\frac{1+\sigma}{2}}
\end{array}\right)
$$

with

$$
\Theta\left(k_{z}, \Delta\right)=\frac{\left(q_{0}^{2} A^{2}+16 \Delta^{2} \cos ^{2} \theta_{0} k_{z}^{2}\right)^{1 / 2}-A q_{0}}{4 \Delta \cos \theta_{0} k_{z}} .
$$

Notice that $\lim _{\Delta \rightarrow 0} \Theta\left(k_{z}, \Delta\right)=0$.

The spectrum of $K^{(0)}$ depends on $\Delta$ through $\Delta^{2}$ and is thus invariant under the exchange of $\Delta$ by $-\Delta$.

\section{APPENDIX C: THE FUNCTIONS $I_{n}(\Delta)$}

The evaluation of the $I_{n}(\Delta)$ functions defined by Eqs. (26) and (27) involves integrals over the wave number $\vec{k}$ that are ultraviolet divergent and thus a short distance cutoff, $\Lambda$, has to be introduced. The cutoff, of course, is naturally provided by the underlying crystal lattice. We find it convenient to use a sharp cylindrical cutoff, so that the spectrum of $K^{(0)}$ is limited to the wave vector region defined by $\left|\vec{k}_{\mathrm{T}}\right|<\Lambda$ and $\left|k_{z}\right|<\Lambda$, where $\vec{k}_{\mathrm{T}}=k_{x} \hat{x}+k_{y} \hat{y}$ is the wave vector projection onto the plane perpendicular to the magnet axis. This cutoff choice is not unreasonable since we are dealing with monoaxial helimagnets.

The function $I_{0}(\Delta)$ is given by the following integral:

$$
I_{0}(\Delta)=\frac{1}{2} \sum_{\sigma= \pm 1} \int \frac{d^{3} k}{(2 \pi)^{3}} \ln \left(\frac{\lambda_{\sigma}(\vec{k})}{k^{2}}\right) .
$$

The integral in $\vec{k}_{\mathrm{T}}$ can be readily performed, and it remains an integral in $k_{z}$ that can be performed numerically. The integral is linearly divergent with the cutoff, and its leading term as $\Lambda \rightarrow \infty$ is

$$
I_{0}(\Delta) \sim \frac{q_{0}^{3}}{8 \pi^{2}}\left[(\ln 2+\pi / 2) A+\pi \Delta^{2} \cos ^{2} \theta_{0}\right] \frac{\Lambda}{q_{0}} .
$$

The function $\Upsilon(t, h)$ involves $I_{0}^{\prime \prime}(0)$, which, to leading order in $\Lambda$, can be obtained from $(\mathrm{C} 1)$ :

$$
I_{0}^{\prime \prime}(0) \sim-\frac{q_{0}^{3}}{4 \pi^{2}}\left[\ln 2+\frac{\pi}{2}+\left(\ln 2+\frac{3 \pi}{2}\right) \frac{h^{2}}{h_{\mathrm{c}}^{2}}\right] \frac{\Lambda}{q_{0}} .
$$

To evaluate $I_{1}$ and $I_{2}$ we need the matrix elements of $Q$ between the eigenstates of $K^{(0)}$. They read

$$
\begin{aligned}
& \left\langle\xi^{\sigma^{\prime}}\left(\vec{k}^{\prime}\right)|Q| \xi^{\sigma}(\vec{k})\right\rangle \\
& \quad=-i C \sum_{\alpha \beta} \epsilon_{\alpha \beta} \phi_{\alpha}^{\sigma^{\prime}}\left(k_{z}^{\prime}\right)^{*} \phi_{\beta}^{\sigma}\left(k_{z}\right)\left[k_{-} \delta_{\vec{k}^{\prime}, \vec{k}+q \hat{z}}+k_{+} \delta_{\vec{k}^{\prime}, \vec{k}-q \hat{z}}\right],
\end{aligned}
$$

where $C=q_{0} \sin \theta_{0}$ and $k_{ \pm}=k_{x} \pm i k_{y}$. The diagonal elements vanish since $q>0$ in the region of stability of the conical state. Therefore, $I_{1}(\Delta)=0$.

Inserting a resolution of the identity in terms of the eigenvalues of $K^{(0)}$ into the definition of $I_{2}(\Delta)$, we get

$$
I_{2}(\Delta)=\frac{1}{4 \rho_{\mathrm{T}}^{2} V} \sum_{\sigma \sigma^{\prime}} \sum_{\vec{k}, \vec{k}^{\prime}} \frac{\left|\left\langle\xi^{\sigma^{\prime}}\left(\vec{k}^{\prime}\right)|Q| \xi^{\sigma}(\vec{k})\right\rangle\right|^{2}}{\lambda_{\sigma^{\prime}}\left(\vec{k}^{\prime}\right) \lambda_{\sigma}(\vec{k})} .
$$

Using (C4) and taking the infinite-volume limit we get

$$
I_{2}(\Delta)=-\frac{C^{2}}{4} \sum_{\sigma= \pm 1} \int \frac{d^{3} k}{(2 \pi)^{3}} k_{\mathrm{T}}^{2} \operatorname{Tr}\left[G(\vec{k}) \tau_{y} G(\vec{k}+\sigma q \hat{z}) \tau_{y}\right]
$$

where $\tau_{y}$ is the Pauli matrix and the matrix $G$ is defined by

$$
G_{\alpha \beta}(\vec{k})=\sum_{\sigma= \pm 1} \frac{\phi_{\alpha}^{\sigma}\left(k_{z}\right) \phi_{\beta}^{\sigma}\left(k_{z}\right)^{*}}{\lambda_{\sigma}(\vec{k})} .
$$

The integral (C6) is complicated, but actually we are only interested in the derivative of $I_{2}(\Delta)$ at $\Delta=0$, which enters the function $\Upsilon(t, h)$, and this is much simpler. It is not difficult 
to see that all the $\Delta$ dependence on the right-hand side of Eq. (C6) is through $\Delta^{2}$, except for the dependence via $q=$ $q_{0}\left(\rho_{z}+\Delta\right)$. Hence, we only need to compute the derivative of $G_{\alpha \beta}$ with respect to $k_{z}$. Taking into account the relations

$$
\begin{gathered}
\lim _{\Delta \rightarrow 0} G_{11}=\left(k^{2}+q_{0}^{2} A_{0}\right)^{-1}, \\
\lim _{\Delta \rightarrow 0} G_{22}=k^{-2}, \\
\lim _{\Delta \rightarrow 0} G_{12}=0, \\
\lim _{\Delta \rightarrow 0} \partial G_{11} / \partial k_{z}=-2 k_{z}\left(k^{2}+q_{0}^{2} A_{0}\right)^{-2}, \\
\lim _{\Delta \rightarrow 0} \partial G_{22} / \partial k_{z}=-2 k_{z} k^{-4}, \\
\lim _{\Delta \rightarrow 0} \partial G_{12} / \partial k_{z}=0,
\end{gathered}
$$

where $A_{0}$ is the value of $A$ at $\Delta=0$, we obtain

$$
\begin{aligned}
I_{2}^{\prime}(0)= & \frac{q_{0}^{3} A_{0}}{4 h_{c}} \int \frac{d^{3} k}{(2 \pi)^{3}} k_{\mathrm{T}}^{2}\left[\frac{1}{k^{2}+q_{0}^{2} A_{0}} \frac{2\left(k_{z}-q_{0}\right)}{\left[\left(\vec{k}-q_{0} \hat{z}\right)^{2}\right]^{2}}+\frac{1}{k^{2}}\right. \\
& \left.\times \frac{2\left(k_{z}-q_{0}\right)}{\left[\left(\vec{k}-q_{0} \hat{z}\right)^{2}+q_{0}^{2} A_{0}\right]^{2}}-\left(q_{0} \rightarrow-q_{0}\right)\right] . \quad(\mathrm{C} 14)
\end{aligned}
$$

The integral over $\vec{k}_{\mathrm{T}}$ can be readily performed and the following integral over $k_{z}$ remains:

$$
\begin{aligned}
I_{2}^{\prime}(0)= & \frac{q_{0}^{3} A_{0}}{8 \pi^{2} h_{c}} \int_{-\Lambda}^{\Lambda} d k_{z}\left(k_{z}-q_{0}\right)\left\{J\left[k_{z}^{2}+q_{0}^{2} A_{0},\left(k_{z}-q_{0}\right)^{2}\right]\right. \\
& \left.+J\left[k_{z}^{2},\left(k_{z}-q_{0}\right)^{2}+q_{0}^{2} A_{0}\right]\right\},
\end{aligned}
$$

where

$$
\begin{aligned}
J\left(E_{1}, E_{2}\right)= & \frac{E_{1}}{\left(E_{2}-E_{1}\right)^{2}} \ln \frac{E_{1}\left(\Lambda^{2}+E_{2}\right)}{E_{2}\left(\Lambda^{2}+E_{1}\right)} \\
& +\frac{\Lambda^{2}}{\left(E_{2}-E_{1}\right)\left(\Lambda^{2}+E_{2}\right)} .
\end{aligned}
$$

The function $J\left(E_{1}, E_{2}\right)$ is analytic at $E_{1}=E_{2}$. The integral (C15) is ultraviolet finite. The logarithmic divergence coming from the integration region $k_{z} \sim \Lambda$ which cancels exactly with the divergence coming from the $k_{z} \sim-\Lambda$ region. It has been evaluated numerically using for $\Lambda$ the same value as in the computation of $I_{0}(\Delta)$ and $I_{0}^{\prime \prime}(0)$.
[1] I. Dzyaloshinskii, J. Phys. Chem. Solids 4, 241 (1958).

[2] T. Moriya, Phys. Rev. 120, 91 (1960).

[3] I. Dzyaloshinskii, Zh. Eksp. Teor. Fiz. 46, 1420 (1964) [Sov. Phys. JETP 19, 960 (1964)].

[4] I. Dzyaloshinskii, Zh. Eksp. Teor. Fiz. 47, 336 (1965) [Sov. Phys. JETP 20, 223 (1965)].

[5] I. Dzyaloshinskii, Zh. Eksp. Teor. Fiz. 47, 992 (1964) [Sov. Phys. JETP 20, 665 (1965)].

[6] Y. Izyumov, Sov. Phys. Usp. 27, 845 (1984).

[7] A. Bogdanov and A. Hubert, J. Magn. Magn. Mater. 138, 255 (1994).

[8] T. Moriya and T. Miyadai, Solid State Commun. 42, 209 (1982).

[9] T. Miyadai, K. Kikuchi, H. Kondo, S. Sakka, M. Arai, and Y. Ishikawa, J. Phys. Soc. Jpn. 52, 1394 (1983).

[10] Y. Togawa, Y. Kousaka, S. Nishihara, K. Inoue, J. Akimitsu, A. S. Ovchinnikov, and J. Kishine, Phys. Rev. Lett. 111, 197204 (2013).

[11] S. Mühlbauer, B. Binz, F. Jonietz, C. Pfleiderer, A. Rosch, A. Neubauer, R. Georgii, and P. Böni, Science 323, 915 (2009).

[12] X. Yu, Y. Onose, N. Kanazawa, J. Park, J. Han, Y. Matsui, N. Nagaosa, and Y. Tokura, Nature (London) 465, 901 (2010).

[13] A. Fert, V. Cross, and J. Sampaio, Nat. Nanotechnol. 8, 152 (2013).

[14] Y. Togawa, Y. Kousaka, K. Inoue, and J. Kishine, J. Phys. Soc. Jpn. 85, 112001 (2016).

[15] Y. Masaki, R. Aoki, Y. Togawa, and Y. Kato, Phys. Rev. B 98, 100402(R) (2018).

[16] H. Han, L. Zhang, D. Sapkota, N. Hao, L. Ling, H. Du, L. Pi, C. Zhang, D. G. Mandrus, and Y. Zhang, Phys. Rev. B 96, 094439 (2017).

[17] J. I. Yonemura, Y. Shimamoto, T. Kida, D. Yoshizawa, Y. Kousaka, S. Nishihara, F. J. T. Goncalves, J. Akimitsu, K. Inoue, M. Hagiwara, and Y. Togawa, Phys. Rev. B 96, 184423 (2017).
[18] F. J. T. Goncalves, T. Sogo, Y. Shimamoto, Y. Kousaka, J. Akimitsu, S. Nishihara, K. Inoue, D. Yoshizawa, M. Hagiwara, M. Mito, R. L. Stamps, I. G. Bostrem, V. E. Sinitsyn, A. S. Ovchinnikov, J. Kishine, and Y. Togawa, Phys. Rev. B 95, 104415 (2017).

[19] V. Laliena, J. Campo, and Y. Kousaka, Phys. Rev. B 95, 224410 (2017).

[20] V. Laliena, J. Campo, and Y. Kousaka, Phys. Rev. B 94, 094439 (2016).

[21] M. Shinozaki, S. Hoshino, Y. Masaki, J. Kishine, and Y. Kato, J. Phys. Soc. Jpn. 85, 074710 (2016).

[22] Y. Nishikawa and K. Hukushima, Phys. Rev. B 94, 064428 (2016).

[23] V. Laliena, J. Campo, J. I. Kishine, A. S. Ovchinnikov, Y. Togawa, Y. Kousaka, and K. Inoue, Phys. Rev. B 93, 134424 (2016).

[24] Y. Masaki and R. L. Stamps, Phys. Rev. B 95, 024418 (2017).

[25] K. Tsuruta, M. Mito, H. Deguchi, J. Kishine, Y. Kousaka, J. Akimitsu, and K. Inoue, Phys. Rev. B 93, 104402 (2016).

[26] N. Sirica, S.-K. Mo, F. Bondino, I. Pis, S. Nappini, P. Vilmercati, J. Yi, Z. Gai, P. C. Snijders, P. K. Das, I. Vobornik, N. Ghimire, M. R. Koehler, L. Li, D. Sapkota, D. S. Parker, D. G. Mandrus, and N. Mannella, Phys. Rev. B 94, 075141 (2016).

[27] M. Garst, J. Waizner, and D. Grundler, J. Phys. D: Appl. Phys. 50, 293002 (2017).

[28] S. Mankovsky, S. Polesya, H. Ebert, and W. Bensch, Phys. Rev. B 94, 184430 (2016).

[29] J. I. Kishine, I. Proskurin, I. G. Bostrem, A. S. Ovchinnikov, and V. E. Sinitsyn, Phys. Rev. B 93, 054403 (2016).

[30] Y. Togawa, T. Koyama, Y. Nishimori, Y. Matsumoto, S. McVitie, D. McGrouther, R. L. Stamps, Y. Kousaka, J. 
Akimitsu, S. Nishihara, K. Inoue, I. G. Bostrem, V. E. Sinitsyn, A. S. Ovchinnikov, and J. Kishine, Phys. Rev. B 92, 220412(R) (2015).

[31] A. C. Bornstein, B. J. Chapman, N. J. Ghimire, D. G. Mandrus, D. S. Parker, and M. Lee, Phys. Rev. B 91, 184401 (2015).

[32] V. Dyadkin, F. Mushenok, A. Bosak, D. Menzel, S. Grigoriev, P. Pattison, and D. Chernyshov, Phys. Rev. B 91, 184205 (2015).

[33] J. I. Kishine, I. G. Bostrem, A. S. Ovchinnikov, and V. E. Sinitsyn, Phys. Rev. B 89, 014419 (2014).

[34] B. Chapman, A. Bornstein, N. Ghimire, D. Mandrus, and M. Lee, Appl. Phys. Lett. 105, 072405 (2014).

[35] N. J. Ghimire, M. A. McGuire, D. S. Parker, B. Sipos, S. Tang, J.-Q. Yan, B. C. Sales, and D. Mandrus, Phys. Rev. B 87, 104403 (2013).

[36] Y. Togawa, T. Koyama, K. Takayanagi, S. Mori, Y. Kousaka, J. Akimitsu, S. Nishihara, K. Inoue, A. Ovchinnikov, and J. Kishine, Phys. Rev. Lett. 108, 107202 (2012).
[37] T. Matsumura, Y. Kita, K. Kubo, Y. Yoshikawa, S. Michimura, T. Inami, Y. Kousaka, K. Inoue, and S. Ohara, J. Phys. Soc. Jpn. 86, 124702 (2017).

[38] R. Aoki, Y. Togawa, and S. Ohara, Phys. Rev. B 97, 214414 (2018).

[39] S. Okumura, Y. Kato, and Y. Motome, J. Phys. Soc. Jpn. 87, 033708 (2018).

[40] V. Laliena and J. Campo, Phys. Rev. B 96, 134420 (2017).

[41] B. Schaub and D. Mukamel, Phys. Rev. B 32, 6385 (1985).

[42] J. Zinn-Justin, Quantum Field Theory and Critical Phenomena (Oxford University Press, New York, 1997).

[43] C. P. Burgess, Phys. Rep. 330, 193 (2000).

[44] S. Coleman and E. Weinberg, Phys. Rev. D 7, 1888 (1973).

[45] B. I. Halperin, T. Lubensky, and S. K. Ma, Phys. Rev. Lett. 32, 292 (1974).

[46] S. Brazovskii, Zh. Eksp. Teor. Fiz. 68, 175 (1975) [JETP 41, 85 (1975)].

[47] M. Janoschek, M. Garst, A. Bauer, P. Krautscheid, R. Georgii, P. Böni, and C. Pfleiderer, Phys. Rev. B 87, 134407 (2013). 